\section{Structural and morphological studies of the dipeptide based L-Pro-L-Val organocatalytic gels and their rheological behaviour $\dagger$}

\author{
Received 20th March 2012, Accepted 8th June 2012 \\ DOI: $10.1039 / \mathrm{c} 2 \mathrm{sm} 25647 \mathrm{a}$

\begin{abstract}
Organocatalytic gels based on the dipeptide sequence L-Pro-L-Val have been studied by two different FTIR techniques. This suggests a different arrangement of the gelator molecules in the self-assembled fibers depending on the organic solvent employed. In acetonitrile and nitromethane the structure of the supramolecular aggregates is similar and provides similar catalytic properties (supramolecular enhancement of basicity). In contrast, the self-assembled fibers obtained in toluene clearly presented a different molecular arrangement consistent with its different catalytic behaviour (enamine-based catalysis). In addition these gels have been studied by microscopy and rheology.
\end{abstract}

Francisco Rodríguez-Llansola, ${ }^{a}$ Beatriu Escuder, ${ }^{* a}$ Ian W. Hamley, ${ }^{b}$ Wayne Hayes ${ }^{b}$ and Juan F. Miravet ${ }^{* a}$

\section{A. Introduction}

Supramolecular gels are materials formed by the one-dimensional assembly of low molecular weight compounds. In these materials, the gelator molecules are able to interact precisely with others via non-covalent interactions (directional in nature such as hydrogen bonding or $\pi-\pi$ stacking or directionally non-specific such as hydrophobic interactions) yielding fibrillar assemblies. ${ }^{1,2}$ The crosslinking of these elongated assemblies produces the formation of a three-dimensional network that is able to trap the solvent. These materials offer unique chemical properties that are related to their supramolecular nature (including reversibility, sensitivity to external stimuli, self-healing ability, etc.). The noncovalent nature of these supramolecular gels has attracted the attention of the scientific community and in recent years several functional materials based on supramolecular gels have been reported. For instance supramolecular gels with application as biomaterials, smart materials and as templates for nanostructured materials have been described. ${ }^{3}$

One of the less studied applications of supramolecular gels is in catalysis, where to date only a limited number of systems have been reported. ${ }^{4}$ Supramolecular gels can be considered as intermediates between homogeneous and heterogeneous catalysts and shares some advantages from both, for example, a high active surface like in a porous solid along with precise control of the structure of the catalytic moiety. Recently, research has focussed on catalytic metallogels but organocatalysis in supramolecular gels remained as an unexplored area. In fact, aggregation is often avoided in organocatalysis because the catalytic sites could be modified upon interaction with other molecules, thus decreasing their reactivity or stereoselectivity. ${ }^{5}$ Nevertheless, there are a few reported examples in which the self-assembly of the organocatalyst serves to improve its catalytic properties. ${ }^{6}$ For example Inoue described that the aggregation of a peptide-based catalyst notably improved the stereoselectivity of the asymmetric addition of hydrogen cyanide to $m$-phenoxybenzaldehyde. ${ }^{6 a-c}$ This precedent prompted us to design organocatalytic gelators and to study their catalytic properties in solution as well as in the gel phase.

Organogelators 1a-c were designed and prepared with L-prolyl groups at both ends of the molecule. L-Pro was chosen as the functional group as a result of its well-known use as a stereoselective organocatalyst for enamine-based reactions. ${ }^{7}$ A L-Val residue was introduced in the gelator design as the assembling fragment, promoting the one-dimensional assembly through hydrogen-bonding between the amide groups (Fig. 1). This assembling fragment has been extensively employed in our group to design functional organogelators with a general bola-amphiphilic structure. $^{8}$

Compounds 1a-c were able to form gels in different organic solvents at low concentrations (see Table S1 in the ESI $\dagger$ ). One of the most interesting properties of these gels is the dramatic effect that the solvent had in the catalytic properties. While gels of 1a-c in acetonitrile and nitroalkanes exhibited basic catalytic

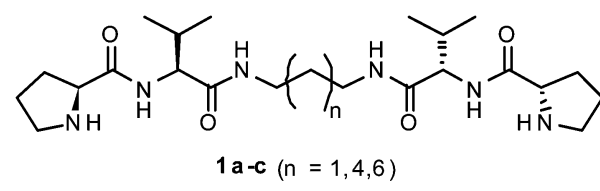

Fig. 1 Chemical structure of organogelators 1a-c.

${ }^{a}$ Departament de Química Inorgànica i Orgànica, Universitat Jaume I, 12071 Castelló, Spain. E-mail: miravet@uji.es; Fax: +34 964728214; Tel: +34964729154

${ }^{b}$ Department of Chemistry, University of Reading, Whiteknights, Reading, UK RG66AD.E-mail: w.c.hayes@reading.ac.uk

$\dagger$ Electronic supplementary information (ESI) available: Additional data on spectroscopy, microscopy and rheology. See DOI: $10.1039 / \mathrm{c} 2 \mathrm{sm} 25647 \mathrm{a}$ 
properties, ${ }^{9}$ the gels in toluene revealed enamine-type catalytic activity. ${ }^{10}$

In this paper, we report an exhaustive analysis of the structure of these gel systems in order to understand the different catalytic properties. First, we will discuss the results obtained via infrared spectroscopy in order to gain a better knowledge of the selfassembly properties of these gelators. In addition the nanoscopic and microscopic aspect of these gels have been characterized by optical and electron microscopy and their macroscopic behaviour quantified by rheology.

\section{B. Experimental section}

\section{Materials}

The synthesis and properties of gelators 1a-c have been previously described. ${ }^{11}$ The basic chemical structure of the molecules used in this study is shown in Fig. 1. The organic solvents used for this investigation were acetonitrile, nitromethane, nitroethane and toluene. All solvents of high purity grade were purchased from Aldrich and were used without further purification.

\section{Techniques}

I. FTIR spectroscopy. Spectra were recorded using a NexusFTIR spectrometer equipped with a DTGS detector and a multiple reflection ATR system. Transmission FTIR measurements were performed using a $\mathrm{CaF}_{2}$ plate, while grazing angle FTIR experiments were done using a gold plate. For both transmission and grazing angle configurations, small aliquots of gels $\left(1.5 \mathrm{wt}^{\%} \%\right)$ were deposited on the corresponding plate and allowed to dry, providing a solid film of dry gel (xerogel). Spectra were scanned 64 times and 128 times for transmission and grazing angle geometries respectively.

II. Cryo-SEM. Experiments were performed using a FEI Quanta FEG 600 Scanning Electron Microscope which is equipped with a Quorum PP2000T Cryo Stage. Usually a small quantity of a gel sample was placed on a copper grid and then it was cooled in nitrogen at $-210{ }^{\circ} \mathrm{C}$ under high vacuum. After the frozen sample was transferred into the cryo stage $\left(-140^{\circ} \mathrm{C}\right)$, it was broken with a knife and sublimed at $\left(-90^{\circ} \mathrm{C}\right)$ for 10 minutes. Then the temperature decreased to $-140^{\circ} \mathrm{C}$ and the sample was coated with Pt at $10 \mathrm{~mA}$ for $20 \mathrm{~s}$ (the argon pressure was $4 \times 10^{-2}$ mbar). Finally the sample was transferred to the microscope.

III. Polarized optical microscopy. POM was performed using an Olympus CX-41 polarized microscope. The samples were prepared in screw-capped vials. After gelation and stabilization at a given temperature, a small amount of gel $(30 \mathrm{mM})$ was placed on a glass cell. No differences were found in the POM images after the film formation. The solvated films of gel were then studied.

IV. Rheology. The rheological properties of the systems were determined using a controlled stress AR-2000 rheometer from TA Instruments. A cone and plate geometry $(10 \mathrm{~mm}$ diameter, gap of $26 \mu \mathrm{m}$ ) was used for all samples. Frequency sweeps were performed in the angular frequency range $0.1-100 \mathrm{rad} \mathrm{s}^{-1}$ with the instrument in oscillatory mode at $25^{\circ} \mathrm{C}$. To ensure that the moduli were independent of strain, preliminary strain sweeps were performed for each sample.

\section{Results and discussion}

\section{FTIR spectroscopic studies}

The xerogels of compounds 1a-c were investigated using FTIR spectroscopy. These gelators are formed by the peptide sequence L-Val-L-Pro and thus most informative frequency regions are related to the corresponding amide groups: (i) $3500-3200 \mathrm{~cm}^{-1}$, corresponding to the $\mathrm{N}-\mathrm{H}$ stretching vibration (amide A), (ii) $1800-1600 \mathrm{~cm}^{-1}$ corresponding to the $\mathrm{C}=\mathrm{O}$ stretching vibrations of the amide groups (amide I) and (iii) $1500-1570 \mathrm{~cm}^{-1}$ associated primarily to the out of plane bending of the $\mathrm{NH}$ unit of the amide groups (amide II). As evident in Table S2 in the ESI $\dagger$, in all cases values around $3500-3400 \mathrm{~cm}^{-1}$ were found for the $\mathrm{N}-\mathrm{H}$ stretching vibration, suggesting that the $\mathrm{N}-\mathrm{H}$ units are in a hydrogen-bonded state. The values found for the amide I band for these xerogels are in the range of $1636-1641 \mathrm{~cm}^{-1}$ and for amide II, values in the range of $1539-1566 \mathrm{~cm}^{-1}$ were found. These frequency values suggest a model of aggregation similar to the $\beta$-sheet arrangement found in peptides. ${ }^{12}$ This model of selfassembly is in agreement with the aggregation models proposed previously for gelators $\mathbf{1 a}-\mathbf{c}^{\mathbf{1 0 , 1 1}}$

The comparison of transmission (T) and grazing incidence (GI) FTIR data has already been used by Hartgerink and coworkers in order to determine the orientation of the intermolecular hydrogen bonding with respect to the main axis of the fibril in peptide amphiphiles with $\beta$-sheet arrangements. ${ }^{13 a}$ The main differences between these two experiments is the angle of incidence of the incoming IR laser beam with respect to the sample $\left(90^{\circ}\right.$ for transmission mode and $10^{\circ}$ for GI mode) and the surface where the sample is deposited $\left(\mathrm{CaF}_{2}\right.$ for $\mathrm{T}$ and gold for GI mode). Comparing the relative intensity of amide I and amide II obtained by the two different FTIR techniques, it is possible to determine if the $\beta$-strands are aligned parallel or perpendicular to the main axis of the fibril (model I and model III in Fig. 2).
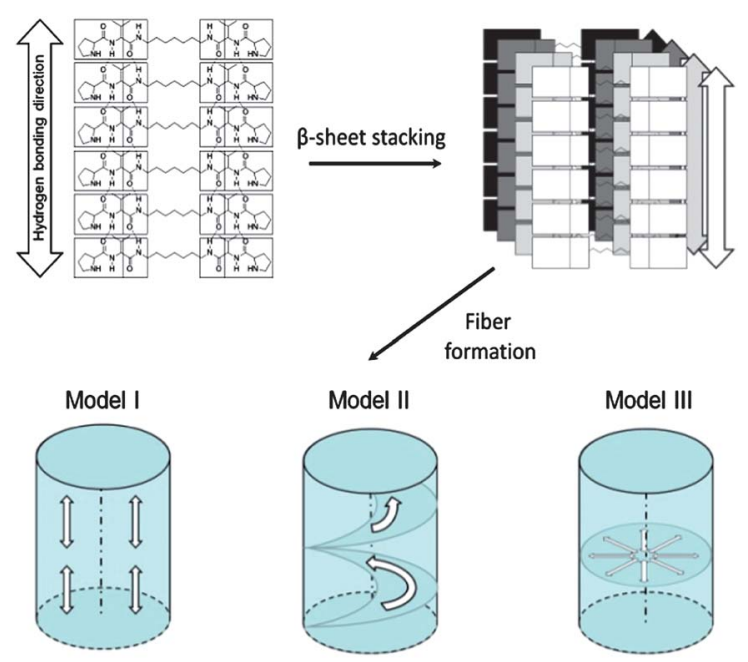

Fig. 2 Models of possible orientation of the hydrogen bonding arrays in self-assembled fibers. 
Intermediate assemblies can also be observed (model II in Fig. 2). These experimental techniques have been employed by several authors in different self-assembling systems ${ }^{13}$ and it can be useful in order to investigate the differences in the self-assembly of gelators 1a-c depending on the solvent employed.

The gels of 1a-c are formed by a three dimensional network of long fibers and they possess a microcrystalline distribution (a high aspect ratio in the fibers and a crystalline nature is required for the study). From a practical viewpoint, a relative decrease of the amide I intensity in GI mode (with respect to the intensity obtained via transmission mode) indicates that the average direction of the $\beta$-sheet is parallel to the axis fiber (Fig. 3A). In the opposite case (the intensity of amide I is higher in GI mode) it can be considered that the average orientation of the intermolecular hydrogen bonding array lies perpendicular to the fiber axis (Fig. 3B).

The results obtained indicate that there are important differences concerning the orientation of the intermolecular hydrogen bonding depending on the solvent employed for organogelators 1a-c (Table 1, Fig. S1 and S2 $\dagger$ ). In acetonitrile gelators 1a and 1c seem to follow model I because a relative decrease in the intensity of the amide I absorbance in GI mode was observed. In the case of compound $\mathbf{1 b}$ clear differences were not observed between the two types of irradiation mode, suggesting a kind of intermediate orientation (for example model II in Fig. 2).

In nitromethane similar results were found. The average orientation of the $\beta$-sheets in the fibers seems to be similar to model I for 1a and $\mathbf{1} \mathbf{c}$ and it is not possible to distinguish a clear fiber model for compound $\mathbf{1 b}$ in this solvent too. Interestingly distinct results were found in toluene (Fig. 3B and $\mathrm{S} 2 \dagger$ ). In this solvent a clear increase of the intensity of amide I in the GI mode was found, suggesting a packing of fibers similar to model III for compounds $\mathbf{1 b}$ and $\mathbf{1 c}$. In this kind of aggregation the $\beta$-sheets are oriented perpendicular to the fiber axis. In the xerogel of
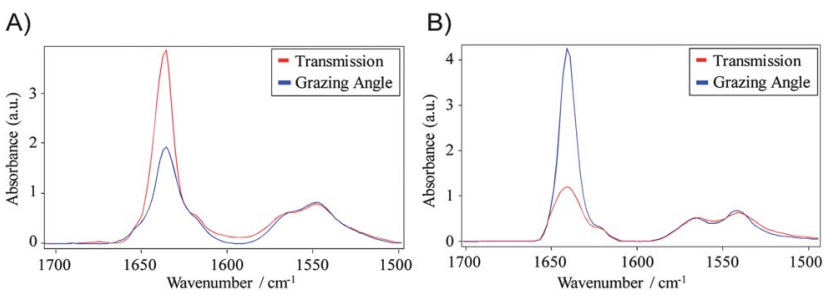

Fig. 3 Transmission $v$ s. grazing angle ATR FTIR spectra for xerogels of compound 1a in acetonitrile (A) and compound $\mathbf{1 b}$ in toluene (B).

Table 1 Orientation models of the hydrogen bonding array in the selfassembled fibers of $\mathbf{1 a}-\mathbf{c}$ in different solvents

\begin{tabular}{cccc}
\hline Entry & Compound & Solvent & Model \\
\hline 1 & $\mathbf{1 a}$ & $\mathrm{CH}_{3} \mathrm{CN}$ & $\mathrm{I}$ \\
2 & $\mathbf{1 b}$ & $\mathrm{CH}_{3} \mathrm{CN}$ & - \\
3 & $\mathbf{1 c}$ & $\mathrm{CH}_{3} \mathrm{CN}$ & $\mathrm{I}$ \\
4 & $\mathbf{1 a}$ & $\mathrm{CH}_{3} \mathrm{NO}_{2}$ & $\mathrm{I}$ \\
5 & $\mathbf{1 b}$ & $\mathrm{CH}_{3} \mathrm{NO}_{2}$ & - \\
6 & $\mathbf{1 c}$ & $\mathrm{CH}_{3} \mathrm{NO}_{2}$ & $\mathrm{I}$ \\
7 & $\mathbf{1 a}$ & Toluene & - \\
8 & $\mathbf{1 b}$ & Toluene & III \\
9 & $\mathbf{1 c}$ & Toluene & III \\
\hline
\end{tabular}

compound 1a a clear difference between the two modes of FTIR experiments was not evident for this solvent. The ability of these compounds to self-assemble in different organic solvents into fibrilar aggregates is associated with their molecular properties (flexible bola-amphiphilic design), allowing different types of molecular distributions into the self-assembled structures.

Therefore, the same average orientation of the hydrogen-bond array and thus a similar arrangement of the molecules 1a-c were observed in acetonitrile and nitromethane. This is in accordance with the similar catalytic properties of the self-assembled fibers found in these two solvents (basic catalysis). The close proximity of the pyrrolidinic residues on the surface of the fibers (that follow model I) and the participation of its $\mathrm{NH}$ in the formation of hydrogen bonds (rigidity of the pyrrolidinic moieties was found in previous HRMAS-NMR studies ${ }^{14}$ ) allow the noncovalent synthesis of a proton relay system, and as a consequence, an enhancement of its basic catalytic properties.

The different orientation of the bola-amphiphilic gelators found in toluene, especially in the case of the organogelator $\mathbf{1 b}$, could explain the different catalytic behaviour of these fibers (enamine based catalysis with enantioselectivity inversion). This model of aggregation seems to give more conformational freedom to the pyrrolidic moieties in the fibers (HRMAS-NMR studies ${ }^{14}$ ) allowing them to react with ketones and to form enamines. The higher mobility of the catalytic groups could explain the absence of a proton relay system in this solvent (an increase of basicity upon gelation was not detected).

It can be concluded that the different structural pattern of the organogelator molecules $\mathbf{1}-\mathbf{c}$ in the one-dimensional aggregates has a remarkable effect on the accessibility and mobility of the catalytic groups and thus into their final catalytic properties.

\section{Microscopic studies}

Microscopy techniques provide insight into the micro and nanostructure for a given gel. Optical microscopy does not require sample handling and allows the observation of the native nature of the aggregates, although it has a low resolution. On the other hand, electron microscopic techniques, i.e. scanning (SEM) and transmission (TEM) electron microscopies have resolutions around $0.2 \mathrm{~nm}$, providing valuable information about the morphology of the aggregates that form the gel. The typical operating conditions for these techniques (high vacuum) require a complete drying of the samples. The process of drying a gel can cause morphological changes in the aspect of their nano-objects due to the phenomena of polymorphism, precipitation or crystallization, and then the images obtained could present artefacts not found in the original gel when it was solvated. ${ }^{15 a}$ One accessible way to investigate the micro and nanostructure of supramolecular gels without these inconveniences involves cryogenic techniques such as cryo-TEM and cryo-SEM. ${ }^{15 b}$ In these methodologies the gels are frozen and afterwards they are carefully lyophilized, keeping the real morphology of the native aggregates. In this process the solvent is eliminated and the solvent pools are converted into cavities. Cryo-TEM provides a higher resolution than cryo-SEM and it is preferred, for example, in the study of the individual supramolecular aggregates that form a gel comprising single nanofibers, nanotubes or nanowires. $^{16}$ In these studies the samples typically present 
concentrations below the minimum gel concentration (MGC) in order to obtain dispersed nano-objects.

For a network of a given supramolecular gel, cryo-SEM is more favourable for different reasons: (1) this imaging technique provides an improved three-dimensional view of the network after lyophilisation; (2) the time required to prepare a sample is significantly lower and (3) it allows the use of different organic solvents with small changes in the preparation method.

I. SEM vs. cryo-SEM studies. To study possible differences between the morphology of the aggregates observed previously by SEM (on xerogels) and those in the solvated state, we studied by cryo-SEM the gels of 1a-c. In Fig. 4 a clear difference between the two microscopic techniques is evident for 1a. In the cryoSEM images, the three-dimensional network of the gel can be seen while in the conventional SEM it is only possible to see the surface obtained after collapse of the fiber network.

In a previous study ${ }^{\mathbf{1 1}}$ we showed that the gelator $\mathbf{1 a}$ forms gels in acetonitrile with different properties as a function of the cooling method employed (spontaneous or controlled cooling). The SEM images obtained showed that the fibers of the xerogel corresponding to the gel obtained by controlled cooling (steps of $5{ }^{\circ} \mathrm{C} \mathrm{min}^{-1}$ ) possess a higher diameter, causing the macroscopic translucent aspect of the gel. In the cryo-SEM study of these gels obtained under the same conditions, it was found that both of them are formed by a fibrillar network. In addition, it can be seen in Fig. 4 that the material obtained by slow cooling seems to present a better defined network of fibres with respect to the kinetically trapped material, in which the network seems more amorphous. In the case of kinetically trapped gel, the fibres are thinner (around $200 \mathrm{~nm}$ in width) than those obtained via controlled conditions (around $600 \mathrm{~nm}$ ). The different size of the fibres explains the different macroscopic aspect of these gels (kinetically trapped gels are transparent and those obtained under controlled conditions are translucent). In addition, it was found that the pore size obtained by the spontaneous cooling method is considerably smaller $(500 \mathrm{~nm})$ as a result of the higher branching phenomena in the gel formation.

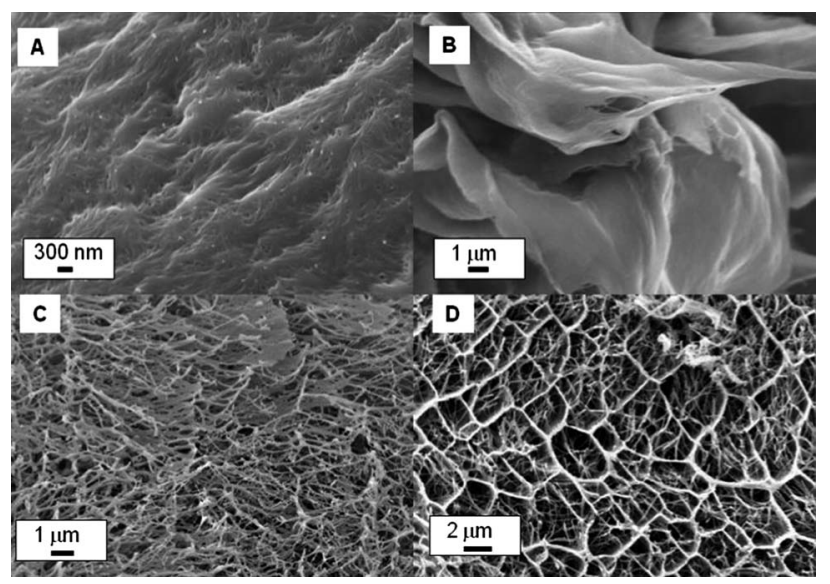

Fig. 4 SEM (top) and cryo-SEM (bottom) images of gels of compound 1a $(32 \mathrm{mM})$ obtained via different gelation protocols. Gels of compound 1a in acetonitrile obtained via spontaneous cooling (left) and by controlled cooling (right).
In the case of gels $\mathbf{1 b}$ and $\mathbf{1 c}$ in nitromethane the morphologies observed by the two techniques were quite similar (Fig. 5A-C). In both cases it is possible to see well defined long fibres but the diameter is considerably higher in the solvated state $(200 \mathrm{~nm}$ by SEM and $800 \mathrm{~nm}$ by cryo-SEM). Furthermore, it can be seen that by changing the time of lyophilization it is possible to change the amount of solvent removed. With a lyophilization of 10 minutes at $-90{ }^{\circ} \mathrm{C}$ it can be seen that only a small quantity of solvent was removed on account of the high boiling point of nitromethane (Fig. 5B). In this case only the surface of the sample is observable. When the time was 15 minutes the quantity of solvent removed was significantly higher, as revealed in the cross-section of the sample (Fig. 5C).

Gels in toluene exhibited several differences between the nanoobjects observed by SEM and cryo-SEM. As evident in Fig. 5 the xerogels of compound $\mathbf{1 b}$ in toluene are comprised of fibres with diameters $\leq 300 \mathrm{~nm}$ in width. In the study of this sample by cryoSEM it was found that the self-assembled network is built by nano-objects with similar length but with a higher thickness. The process of drying in this sample seems to produce fibres in the xerogels which present a lower thickness than in the solvated state.

It can be concluded that in some cases the objects observed previously by SEM in supramolecular gels of $1 \mathbf{a}-\mathbf{c}$ are not totally representative of the real nanostructures that produce the gelation. For this reason cryo-SEM is a more reliable technique to study the three dimensional network of aggregates that form a supramolecular gel.

II. Structure of fibrillar networks. The most accepted mechanism for the formation of a supramolecular gel is nucleationmediated branching or "crystallographic mismatch branching". ${ }^{17}$

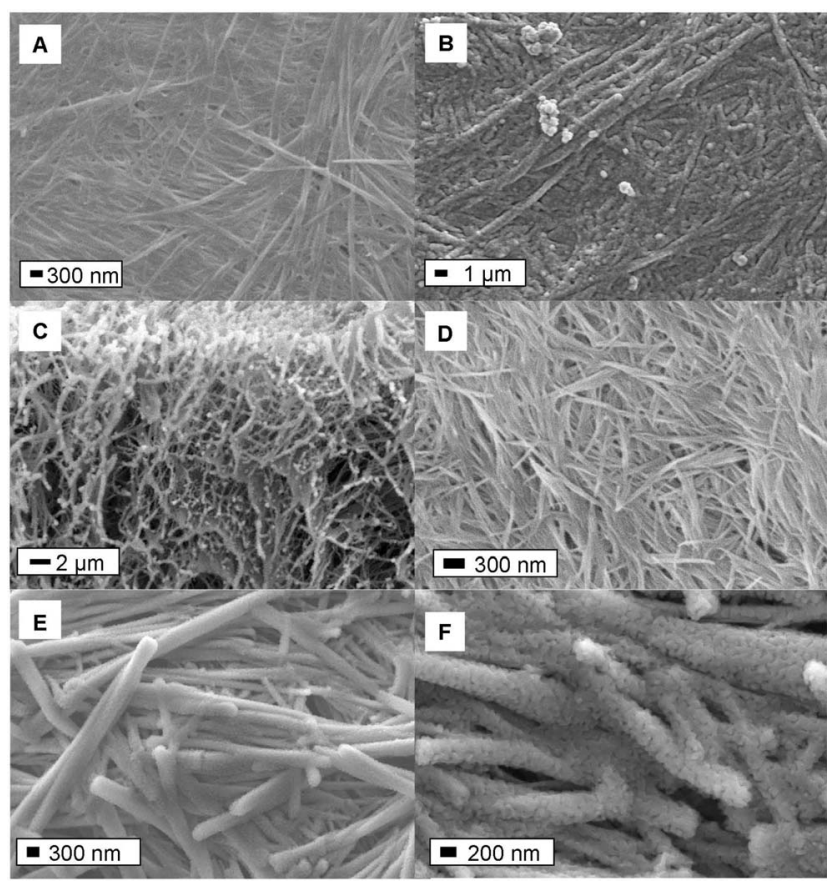

Fig. 5 SEM and cryo-SEM images of gels of compounds $\mathbf{1 b}$ and $\mathbf{1 c}$. Gel of compound 1c $(18 \mathrm{mM})$ in nitromethane observed by SEM (A) and cryo-SEM (B and C). Gel of compound $\mathbf{1 b}(30 \mathrm{mM})$ in toluene observed by SEM (D) and cryo-SEM (E and F). 
According to this mechanism the formation of the threedimensional network initiates from primary nucleation followed by the growth and branching of fibrous arms. The number of starting nuclei and the ratio between fibre growing and branching determines the final properties of a given gel. A fibrillar network can be classified as a "single fibre network" or as a "multidomain fibre network". In a single fibre network, the primary nucleation forms a well interconnected and interlocked 3-D network of fibres. In some cases, when the branching is very compact, the formation of spherulites is observed. If they are much more branched, they will not be able to intercalate with the adjacent spherulites, yielding a multidomain network. The issue of spherulites versus fibrillar network formation in molecular gels has been studied previously, for example, by Weiss. ${ }^{17 e}$

Spherulites are regular birefringent structures with radial symmetry in three dimensions and a circular symmetry in a thin section and can be easily observed by polarized optical microscopy (POM) Fig. $6 .{ }^{18}$ They present characteristic Maltese-cross extinction patterns when observed in a thin section between crossed polarizers, as a result of the coincidence of the mutually perpendicular optical axis of the spherulites with the respective orientations of the polarizer and analyzer. Spherulites are a very common kind of self-assembly observed during polymer crystallization and their formation is well documented for synthetic polymers such as polyethylene. ${ }^{18 b}$ The formation of these semicrystalline objects has been observed in many other systems including natural polymers as, for example, in carbohydrates such as cellulose, ${ }^{19}$ chitin $^{20}$ and chitosan ${ }^{21}$ and in proteins such as bovine insulin. ${ }^{22}$

Keeping in mind that the self-assembly of compounds 1a-c presents a model of aggregation similar to the $\beta$-sheet arrangement in peptides, they were studied by POM in order to obtain
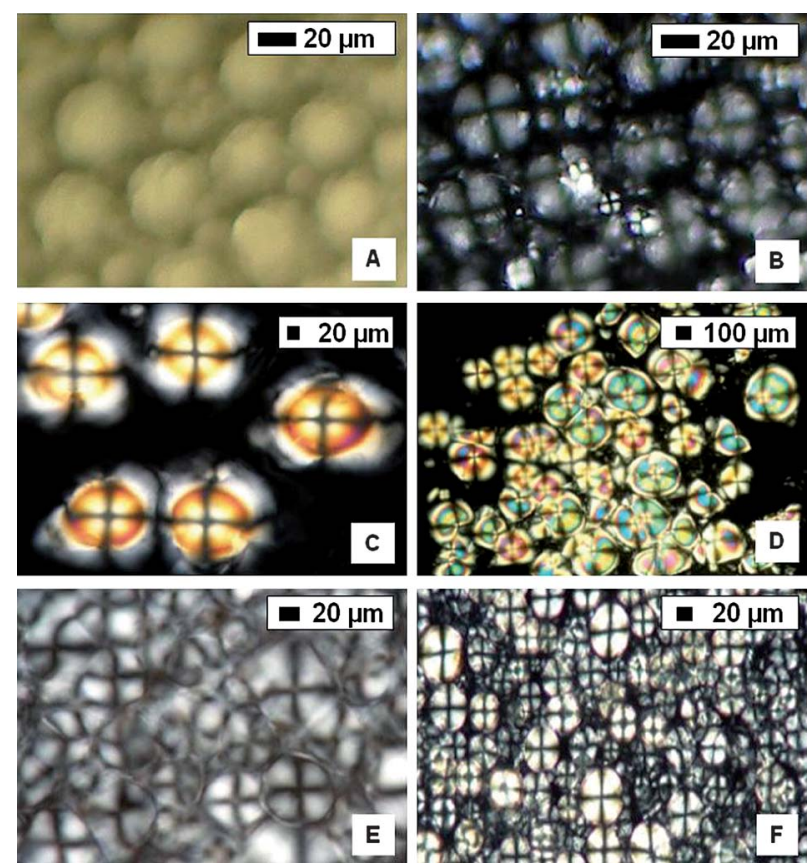

Fig. 6 Optical microscope (A) and POM (B-F) images. (A) Multidomain formed by $1 \mathbf{c}$ in toluene; (B) multidomain 1c in toluene; (C) gel of 1c in nitroethane; (D) gel of 1a in toluene; (E) gel of 1c in toluene; (F) gel of $\mathbf{l b}$ in acetonitrile - spontaneous cooling. insight into the aggregation process and possible spherulite formation.

When the gels of 1a-c in acetonitrile were observed by POM it was found that the gels obtained by controlled cooling presented a crystalline texture, whereas those obtained by spontaneous cooling exhibited significant numbers of spherulites of different sizes (Fig. S3 $\uparrow$ ). This behaviour has been associated with the degree of supersaturation at the initial stage of the nucleationgelation. ${ }^{23}$

In the case of compound 1a it was observed that the gel obtained by controlled cooling comprises an interconnected network with a crystalline texture. In contrast, under conditions of spontaneous cooling it was observed that the gel was formed by individual spherulites with size around $20 \mu \mathrm{m}$. This result can be understood keeping in mind that at low cooling rates crystallographic mismatches are not present yielding long fibres with low branching (as observed by cryo-SEM). Similar results were obtained with compounds $\mathbf{1 b}$ and $\mathbf{1 c}$ in this solvent. The gels obtained via spontaneous cooling yielded materials with a higher number of spherulites than those obtained via the controlled cooling method.

The gels formed in toluene by $\mathbf{1 a - c}$ presented different textures depending on the gelator (Fig. S4 $\dagger$ ). In the case of compound 1a coloured spherulites with diameter around $2 \mu \mathrm{m}$ were found. These colour phenomena are associated with a different orientation of the molecules within the spherulite, modifying its refractive index. ${ }^{24}$ The POM texture suggests a multidomain network formed by a high number of individual spherulites. In the case of the gels of compound $\mathbf{1} \mathbf{c}$ in this solvent it was observed that the network is formed by individual spherulites too, but the size of them is considerably lower $(50 \mu \mathrm{m})$. In the case of compound $\mathbf{1 b}$ only some dispersed spherulites were observed and a more crystalline aspect was found. These results are in agreement with the gelation capacity of compounds $\mathbf{1 a - c}$ in this solvent. Compound $\mathbf{1 b}$ presents the lowest MGC in this solvent and forms single fibre network gels with only some small spherulites. Gelators 1a and $\mathbf{1} \mathbf{c}$ formed multidomain networks of spherulites and presented higher MGC values. The tendency of gelators 1a-c to form spherulites was found to be lower in nitroalkanes (Fig. S5 $\dagger$ ). In nitromethane a large number of interconnected small spherulites was found in the case of compound 1a. In the case of the gels of $\mathbf{1 b}$ and $\mathbf{1 c}$, which present a lower MGC in this solvent, the amount of birefringent objects was considerably lower. In nitroethane only some dispersed big spherulites were observed.

It can be concluded that gelators 1a-c present a high tendency to form spherulites as previously detected in the gelation studies in acetonitrile. ${ }^{11}$ It was found that compound $\mathbf{1 c}$ at concentrations below MGC is able to self-assemble into discrete spherulites of around $1 \mathrm{~mm}$ in diameter (Fig. S6 $\dagger$ ). These spherulites presented an amorphous core from where the self-assembled fibres started to grow.

The tendency of compounds 1a-c to form spherulites could be related to their conformational properties. In Fig. 7 a possible mechanism for the formation of a multidomain fibre network or a single fibre network is shown depending on the cooling method employed. In conformational studies it was determined that the amide groups of these compounds are able to interact in an intramolecular fashion yielding folded molecules in solution. ${ }^{10,11}$ 


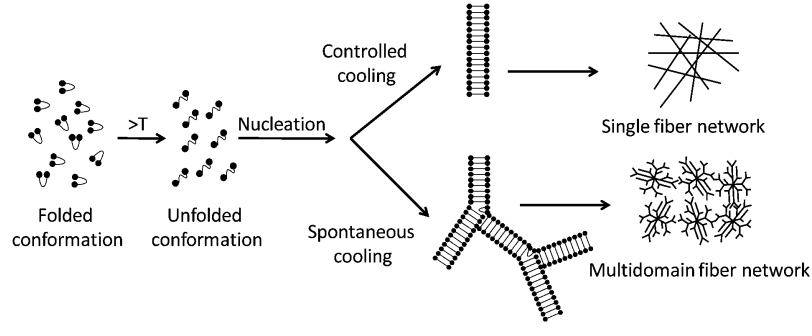

Fig. 7 Illustration of fibre network formation depending on the method of cooling employed.

At high gelator concentrations the molecules switch to an extended conformation and interact with each other through intermolecular hydrogen bonding between the amide groups, yielding one-dimensional aggregates. Thus, the amide groups are bound in an intramolecular or intermolecular fashion depending on the concentration. In a typical gelation process, the gelator is dissolved in a solvent by heating. At high temperatures the intermolecular interactions that fold these molecules in solution are broken and the gelator molecules present an extended conformation. In super-saturated conditions depending on the rate of cooling the amide groups of these molecules will form an intra- or inter-molecular hydrogen bond. The gelator molecules in a folded conformation will present a wrong conformation for one-dimensional assembly and then they will produce a mismatch in the fibrillar assembly, producing, for example, branch phenomena (Fig. 7). If the branching phenomenon is important, spherulites will be formed and a multidomain network will be obtained. In the case of a low mismatch in the one-dimensional assembly, a highly interconnected network of fibres will be obtained.

\section{Rheology}

In a supramolecular gel the macroscopic properties are directly dependent on the microscopic structure of the three-dimensional fiber network. ${ }^{1,25}$ Usually when the concentration of the gelator is close to the MGC the number of fibres and their cross-linking is low, and thus the material is considered as a weak gel. In this situation the presence of the nanofibres increases the viscosity of the solution but it is not enough to prevent the liquid flow. At concentrations higher than the MGC the number and size of the fibres is higher, yielding a cross-linked three dimensional network that is able to immobilize the solvent. It is logical to think that the modulus of a given gel will increase with an increase of the gelator concentration. This is true until a given concentration of the gelator. It was found that at high concentration the macroscopic properties of these materials are inferior. ${ }^{26}$ This behaviour can be ascribed to supersaturation effects (branching, polymorphism, precipitation, defects in the selfassembly process, etc.).

The dynamic shear moduli were measured for gels of $\mathbf{1 a}-\mathbf{c}$ and 2. Gels were subjected to a non-destructive frequency-sweep in which an initial stress of $3 \mathrm{~Pa}$ was allowed to adjust to a constant strain of $0.01 \%$. As an example, it can be seen in Fig. 8 that a gel of $1 \mathbf{a}$ in acetonitrile presented values of $G^{\prime}$ and $G^{\prime \prime}$ virtually independent of the oscillation frequency. The value of $G^{\prime}$ obtained was at least one order of magnitude higher than $G^{\prime \prime}$, revealing the dominant elastic behaviour of these gels. These two rheological characteristics are present in all of the gels studied at moderate concentrations (Fig. S7-S9†).

Studies of $G^{\prime}$ and $G^{\prime \prime} v s$. concentration of the gelator revealed a general increase in both moduli values up to a certain concentration, after which the modulus decreased and the values of $G^{\prime}$ and $G^{\prime \prime}$ became similar, turning the elastic gels into a viscoelastic material. Fig. 8 shows the concentration dependence on the values of $G^{\prime}$ and $G^{\prime \prime}$ of $\mathbf{1 a}$ and $\mathbf{1 c}$ in acetonitrile. It can be seen that at low concentration the values of $G^{\prime}$ and $G^{\prime \prime}$ of compound 1c are higher based on its lower MGC and solubility. A gelator with low solubility (and low values of MGC) presents a low fraction of gelator molecules in solution in contrast with the number of molecules present in the solid state (in the fibres). ${ }^{27}$ Additionally, it can be observed that the values of $G^{\prime}$ and $G^{\prime \prime}$ for 1c achieve a maximum at $35 \mathrm{mM}$ and afterwards there is a smooth decrease in its rheological properties. This behaviour can be understood if one considers the positive effect of an increase in the gelator concentration (higher number and size of fibers) and the negative effect on the mechanical properties associated with the supersaturation effects. The lower solubility of this gelator in acetonitrile suggests that defects in the self-assembly appear before that in the case of gelator 1a.

At concentrations above $40 \mathrm{mM}$ the difference between $G^{\prime}$ and $G^{\prime \prime}$ becomes lower in the case of compound 1c while in the gels of compound 1a it remains equal. This behaviour can be ascribed to the different solubility of these gelators in acetonitrile. Gelator 1c
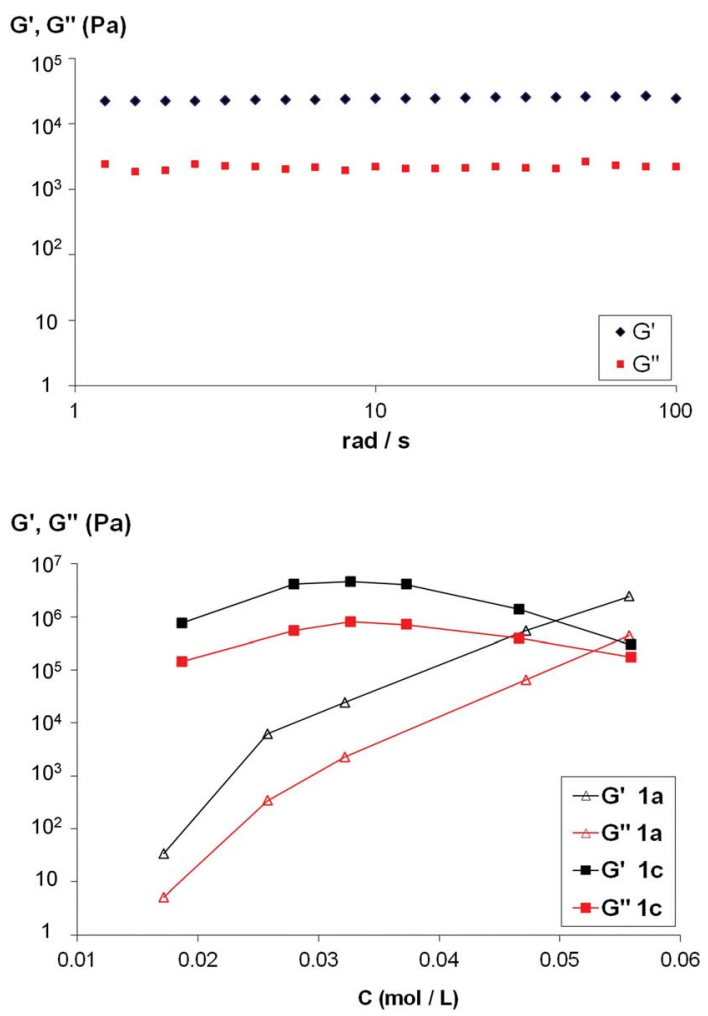

Fig. 8 Frequency sweep for a gel in acetonitrile of compound 1a $(32 \mathrm{mM})$. Representation of the elastic modulus $\left(G^{\prime}\right)$ and viscous modulus $\left(G^{\prime \prime}\right) v s$. the gelator concentration for gels $1 \mathrm{a}$ and $\mathbf{1 c}$ in acetonitrile (spontaneous cooling). 
is able to form well-defined fibres at concentrations lower than 1a but presents a decrease of the macroscopic properties (related to super-saturation phenomena) at a lower concentration.

Similar results were found for gels in nitromethane (Fig. S10†). Gels of compound 1c in this solvent presented better rheological properties with respect to the gels obtained with compound 1a at low concentrations. When the concentration of the gelator was higher than $30 \mathrm{mM}$, the properties of the gels of 1 a become better as a result of the formation of a well cross-linked network of fibres yet worse in the case of $\mathbf{1 c}$ in which the super-saturation phenomena become important.

The effect that the gelation methodology and the presence of co-solvents have on the rheological properties of the final materials was examined. Different gels of 1a in acetonitrile were prepared by spontaneous and controlled cooling methods. It can be seen in Fig. 9 that the properties of the final materials were significantly different. At low concentration the gels obtained by spontaneous cooling presented values for $G^{\prime}$ and $G^{\prime \prime}$ significantly lower than those obtained with the controlled cooling process. As shown by cryo-SEM, the gels obtained by fast cooling presented short and thin fibres with a high degree of branching. The shape and size of these self-assembled fibres obtained by the spontaneous cooling method show that the final rheological properties of the gel are lower with respect to those obtained by the controlled cooling method.
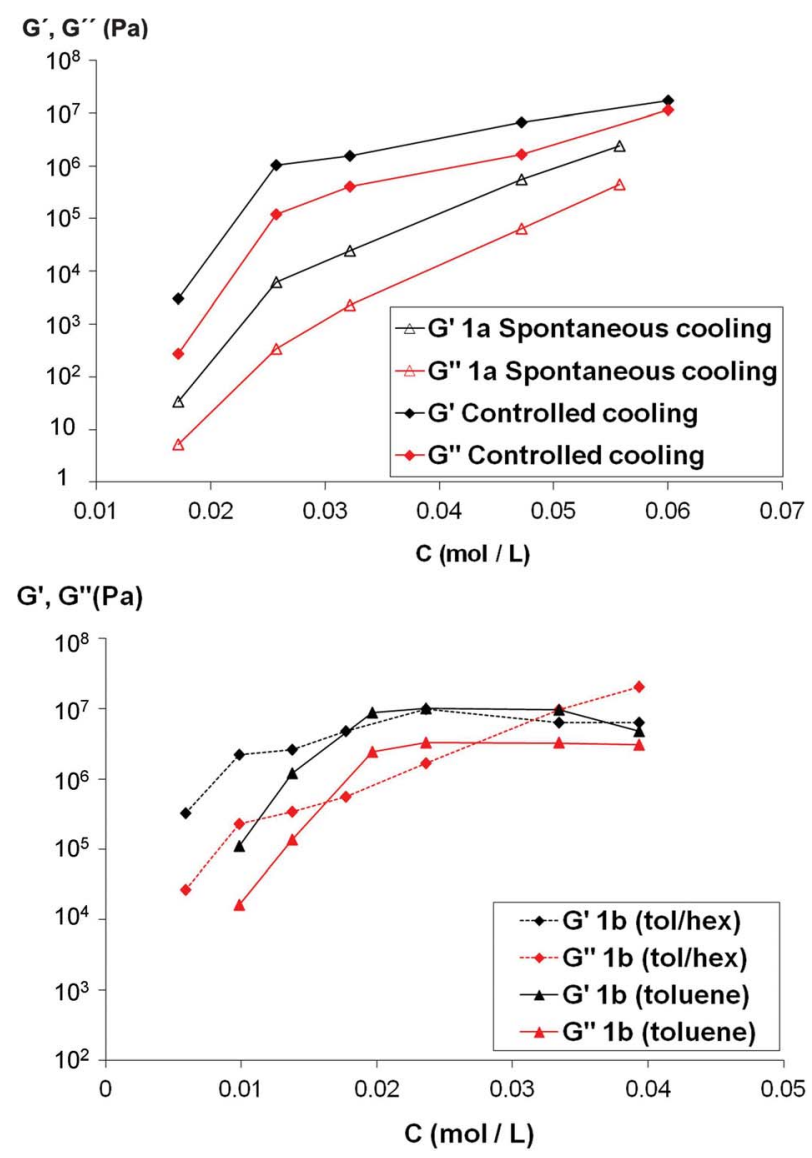

Fig. 9 Representation of the elastic modulus $\left(G^{\prime}\right)$ and viscous modulus $\left(G^{\prime \prime}\right)$ vs. the gelator concentration. (Top) Gels $\mathbf{1 a}$ and $\mathbf{1 c}$ in acetonitrile (spontaneous cooling and controlled cooling). (Bottom) Gel of $\mathbf{1 b}$ in toluene pure and in toluene with $10 \%$ of hexane.
It was found in the gelation studies that the introduction of a small quantity of hexane increased the modulus of the gels of compound 1b in toluene. For example, it was found that the gels formed in a mixture 9/1 toluene-hexane possessed a higher thermal stability and a lower quantity of gelator molecules in solution (lower solubility) than the gels obtained in toluene. ${ }^{10} \mathrm{It}$ can be seen in Fig. 9 that at low concentration the gels obtained in toluene lead to lower shear modulus values with respect to those obtained in the presence of $10 \%$ of hexane. At high concentration the super-saturation effects (i.e. precipitation, branching) are important in the gels obtained in the presence of hexane and then there is a decrease in its modulus.

This study shows how sensitive the rheological properties of these supramolecular materials are to small changes in the concentration of the gelator, gelation conditions and solvent. In addition, it has been shown that the gelators 1a-c are able to form supramolecular gels only in a given concentration range.

\section{Conclusions}

We have studied the gels formed by $\mathbf{1 a}-\mathbf{c}$ by FTIR spectroscopy in transmission and in grazing angle modes. The results obtained suggest aligned self-assembled structures resembling the $\beta$-sheet pattern in peptides. In addition, it was found that the average direction of these $\beta$-sheets with respect to the self-assembled fibre axis is different depending on the solvent. A similar distribution was found in the gels of nitromethane and acetonitrile where the fibres present a supramolecular basicity. In contrast, in toluene a different orientation of the hydrogen bonding was detected. It could explain the non-basic catalytic properties of the selfassembled fibres in this solvent and its ability to form enamines.

These gels were analyzed with cryo-SEM and differences in morphology were noted with respect to those observed previously by regular SEM. The differences achieved can be associated with the phenomena of crystallisation or polymorphism during the solvent elimination process. POM revealed that the gelators 1a-c present a high tendency to form spherulites as a result of their conformational characteristics. In the case of the gels in acetonitrile it was detected that depending on the cooling method employed it is possible to switch the properties of the three-dimensional network. At low cooling rates (controlled cooling) single fibre networks were obtained while if the samples are cooled spontaneously the final network is formed by individual spherulites.

Finally, rheology was used to quantify the effect that the gelator concentration, the gelation method employed and the presence of a co-solvent has in the final macroscopic properties.

We thank Spanish Ministry of Science and Innovation (Grant CTQ2009-13961) and Universitat Jaume I-Bancaixa (Grant P1 1B2009-42) for financial support. FRL thanks Generalitat Valenciana for a FPI fellowship.

\section{Notes and references}

1 (a) Molecular Gels: Materials with Self-Assembled Fibrillar Networks, ed. R. G. Weiss and P. Terech, Springer, Dordrecht, The Netherlands, 2005; (b) Top. Curr. Chem., ed. F. Fages, 2005, vol. 256, p. 1; (c) D. K. Smith, Molecular Gels-Nanostructured Soft Materials in Organic Nanostructures, ed. J. L. Atwood and J. W. Steed, WileyVCH, Weinheim, 2008. 
2 (a) P. Terech and R. G. Weiss, Chem. Rev., 1997, 97, 3133; (b) J. H. van Esch and B. L. Feringa, Angew. Chem., Int. Ed., 2000, 39, 2263; (c) P. Dastidar, Chem. Soc. Rev., 2008, 37, 2699; (d) J. H. van Esch, Langmuir, 2009, 25, 8392.

3 (a) N. M. Sangeetha and U. Maitra, Chem. Soc. Rev., 2005, 34, 821; (b) A. R. Hirst, B. Escuder, J. F. Miravet and D. K. Smith, Angew. Chem., Int. Ed., 2008, 47, 8002; (c) S. Banerjee, R. K. Das and U. Maitra, J. Mater. Chem., 2009, 19, 6649.

4 (a) B. Escuder, F. Rodríguez-Llansola and J. F. Miravet, New J. Chem., 2010, 34, 1044; (b) D. D. Diaz, D. Kuhbeck and R. J. Koopmans, Chem. Soc. Rev., 2011, 40, 427.

5 (a) H. S. Rho, S. H. Oh, J. W. Lee, J. Y. Lee, J. Chin and C. E. Song, Chem. Commun., 2008, 1208; (b) S. H. Oh, H. S. Rho, J. W. Lee, J. E. Lee, S. H. Youk, J. Chin and C. E. Song, Angew. Chem., Int. Ed., 2008, 47, 7872 .

6 (a) K. Tanaka, A. Mori and S. Inoue, J. Org. Chem., 1990, 55, 181; (b) H. Danda, Synlett, 1991, 263; (c) E. A. C. Davie, S. M. Mennen, Y. $\mathrm{Xu}$ and S. J. Miller, Chem. Rev., 2007, 107, 5759; (d) M. O. Guler and S. I. Stupp, J. Am. Chem. Soc., 2007, 129, 12082.

7 (a) B. List, R. A. Lerner and C. F. Barbas III, J. Am. Chem. Soc., 2000, 122, 2395; (b) W. Notz, F. Tanaka and C. F. Barbas III, Acc. Chem. Res., 2004, 37, 580; (c) B. List, Acc. Chem. Res., 2004, 37, 548; (d) B. List, in Modern Aldol Reactions, ed. R. Mahrwald, Wiley-VCH, Weinheim, 2004, vol. 1, pp. 161-200; (e) D. Enders, M. R. M. Hüttl, C. Grondal and G. Raabe, Nature, 2006, 441, 861; (f) C. Palomo and A. Mielgo, Angew. Chem., Int. Ed., 2006, 45, 7876; $(g)$ G. Guillena, C. Nájera and D. J. Ramón, Tetrahedron: Asymmetry, 2007, 18, 2249; (h) C. F. Barbas III, Angew. Chem., Int. Ed., 2008, 47, 42.

8 (a) J. F. Miravet and B. Escuder, Org. Lett., 2005, 7, 4791; (b) J. F. Miravet and B. Escuder, Chem. Commun., 2005, 5796; (c) B. Escuder, S. Martí and J. F. Miravet, Langmuir, 2005, 21, 6776.

9 (a) F. Rodríguez-Llansola, B. Escuder and J. F. Miravet, Org. Biomol.Chem., 2009, 7, 3091; (b) F. Rodríguez-Llansola, B. Escuder and J. F. Miravet, J. Am. Chem. Soc., 2009, 131, 11478.

10 F. Rodríguez-Llansola, J. F. Miravet and B. Escuder, Chem.-Eur. J., $2010,16,8480$.

11 F. Rodriguez-Llansola, J. F. Miravet and B. Escuder, Chem Commun., 2009, 209.

12 (a) A. Aggeli, M. Bell, N. Boden, J. N. Keen, P. F. Knowles, T. C. B. McLeish, M. Pitkeathly and S. E. Radford, Nature, 1997, 386, 259; (b) J. Kubelka and T. A. Keiderling, J. Am. Chem. Soc., 2001, 123, 12048.
13 (a) S. E. Paramonov, H.-W. Jun and J. D. Hartgerink, J. Am. Chem. Soc., 2006, 128, 7291; (b) V. Castelletto, I. W. Hamley, P. J. F. Harris, U. Olsson and N. Spencer, J. Phys. Chem. B, 2009, 113, 9978; (c) E. T. Pashuck, H. Cui and S. I. Stupp, J. Am. Chem. Soc., 2010, 132, 6041; (d) I. F. Gallardo and L. J. Webb, Langmuir, 2010, 26, 18959; (e) N. Schüwer and H. A. Klok, Langmuir, 2011, 27, 4789; (f) M. A. Ramin, G. Le Bourdon, N. Daugey, B. Bennetau, L. Vellutini and T. Buffeteau, Langmuir, 2011, 27, 6076; $(g)$ F. Rodríguez-Llansola, B. Escuder, J. F. Miravet, D. HermidaMerino, I. W. Hamley, C. J. Cardin and W. Hayes, Chem. Commun., 2011, 46, 7960.

14 S. Iqbal, F. Rodriguez-Llansola, B. Escuder, J. F. Miravet, I. Verbruggen and R. Willem, Soft Matter, 2010, 6, 1875.

15 (a) I. A. Coates and D. K. Smith, Chem.-Eur. J., 2009, 15, 6340; (b) M. A. Rogers, A. K. Smith, A. J. Wright and A. G. Marangoni, J. Am. Oil Chem. Soc., 2007, 84, 899.

16 V. Castelletto, I. W. Hamley, C. Cenker, U. Olsson, J. Adamcik, R. Mezzenga, J. F. Miravet, B. Escuder and F. Rodríguez-Llansola, J. Phys. Chem. B, 2011, 115, 2107.

17 (a) P. Terech, D. Pasquier, V. Bordas and C. Rossat, Langmuir, 2000, 16, 4485; (b) X. Y. Liu, P. D. Sawant, W. B. Tan, I. B. M. Noor, C. Pramesti and B. H. Chen, J. Am. Chem. Soc., 2002, 124, 15055; (c) X. Y. Liu and P. D. Sawant, Adv. Mater., 2002, 14, 421; (d) J.-L. Li and X.-Y. Liu, Adv. Funct. Mater., 2010, 20, 3196; (e) X. Huang, S. R. Raghavan, P. Terech and R. G. Weiss, J. Am. Chem. Soc., 2006, 128, 15341.

18 (a) J. H. Magill, J. Mater. Sci., 2001, 36, 3143; (b) D. C. Bassett, J. Macromol. Sci., Part B: Phys., 2003, 42, 227.

19 S. Kobayashi, L. J. Hobson, J. Sakamoto, S. Kimura, J. Sugiyama, T. Imai and T. Itoh, Biomacromolecules, 2000, 1, 168.

20 S. B. Murray and A. C. Neville, Int. J. Biol. Macromol., 1998, 22, 137.

21 S. B. Murray and A. C. Neville, Int. J. Biol. Macromol, 1997, 20, 123.

22 M. R. H. Krebs, C. E. MacPhee, A. F. Miller, I. E. Dunlop, C. M. Dobson and A. M. Donald, Proc. Natl. Acad. Sci. U. S. A., 2004, 101, 14420.

23 R. Wang, X.-Y. Liu, J. Xiong and J. Li, J. Phys. Chem. B, 2006, 110, 7275 .

24 J. Tao, G. Mao and L. Daehne, J. Am. Chem. Soc., 1999, 121, 3475.

25 (a) B. Roy, P. Bairi, A. Saha and A. K. Nandi, Soft Matter, 2011, 7, 8067; (b) P. Bairi, B. Roy and A. K. Nandi, RSC Adv., 2012, 2, 264. 26 F. M. Menger and K. L. Caran, J. Am. Chem. Soc., 2000, 122, 11679.

27 A. R. Hirst, I. A. Coates, T. R. Boucheteau, J. F. Miravet, B. Escuder, V. Castelletto, I. W. Hamley and D. K. Smith, J. Am. Chem. Soc., 2008, 130, 9113 . 